\title{
Tunable Surface Plasmon Polaritons with Monolithic Schottky Diodes
}

DOI:

10.1021/acsaelm.9b00499

\section{Document Version}

Accepted author manuscript

Link to publication record in Manchester Research Explorer

\section{Citation for published version (APA):}

Zhang, Y., Ling, H., Chen, P., Qian, P., Shi, Y., Wang, Y., Feng, H., Xin, Q., Wang, Q., Shi, S., Pan, X., Sheng, X., \& Song, A. (2019). Tunable Surface Plasmon Polaritons with Monolithic Schottky Diodes. ACS Applied Electronic Materials. https://doi.org/10.1021/acsaelm.9b00499

\section{Published in:}

ACS Applied Electronic Materials

\section{Citing this paper}

Please note that where the full-text provided on Manchester Research Explorer is the Author Accepted Manuscript or Proof version this may differ from the final Published version. If citing, it is advised that you check and use the publisher's definitive version.

\section{General rights}

Copyright and moral rights for the publications made accessible in the Research Explorer are retained by the authors and/or other copyright owners and it is a condition of accessing publications that users recognise and abide by the legal requirements associated with these rights.

\section{Takedown policy}

If you believe that this document breaches copyright please refer to the University of Manchester's Takedown Procedures [http://man.ac.uk/04Y6Bo] or contact uml.scholarlycommunications@manchester.ac.uk providing relevant details, so we can investigate your claim.

\section{OPEN ACCESS}




\title{
Tunable Surface Plasmon Polaritons with Monolithic Schottky Di- odes
}

\author{
Yifei Zhang ${ }^{\dagger}$, Haotian Ling ${ }^{\dagger}$, Pingjian Chen ${ }^{\dagger}$, Pengfei Qian ${ }^{\dagger}$, Yanpeng Shi ${ }^{\dagger}$, Yiming Wang ${ }^{\dagger}$, Huayu \\ Feng $^{\dagger}$, Qian Xin ${ }^{\dagger}$, Qingpu Wang ${ }^{\dagger *}$, Shouyuan Shi ${ }^{\ddagger}$, Xiaomin Pan $^{\S}$, Xinqing Sheng ${ }^{\S}$, and Aimin Song ${ }^{\dagger, \|, *}$ \\ ${ }^{\dagger}$ Center of Nanoelectronics and School of Microelectronics, Shandong University, Jinan, China \\ Department of Electrical and Computer Engineering, University of Delaware, Newark, DE, U.S.A. \\ ${ }^{\S}$ School of Information and Electronics, Beijing Institute of Technology, Beijing, China \\ 'School of Electrical and Electronic Engineering, University of Manchester, Manchester, United Kingdom \\ Supporting Information Placeholder
}

\begin{abstract}
Surface plasmon polaritons (SPPs) provide subwavelength electric field confinement ranging from microwave to the visible. Several approaches have been explored to manipulate SPPs with a typical modulation capability of only a few percent. Here, active control of SPPs using monolithically fabricated Schottky diodes has been first designed and realized, achieving a continuous and significant modulation of transmission, reflection, and absorption. The SPPs propagating on a metal line are attenuated by using split ring resonators (SRRs) with an In-Ga-Zn-O Schottky diode bridging each SRR split gap. The resistance of the diodes can be tuned over a range of a few orders of magnitude with bias voltage, which continuously transforms each SRR to a metallic quasi-loop with subdued resonance. A remarkable modulation of $40 \%$ and $19 \%$ was demonstrated for the transmission and absorption, respectively, which to the best of our knowledge has not been achieved before.
\end{abstract}

\section{INTRODUCTION}

Surface plasmon polaritons (SPPs) are propagating surface waves with highly confined electric field at the interface between two different materials with opposite permittivities. ${ }^{1-5}$ With the advantages of non-diffraction limit and strong electric field enhancement, SPPs have found many applications from microwave to optical frequencies, such as super-resolution imaging, miniaturized sensors, photovoltaics, miniaturized integrated circuits etc. ${ }^{5-13}$ SPPs were originally found at metal-dielectric interface in visible and near-infrared spectra due to the plasmonic properties of noble metal and the corresponding negative permittivity. ${ }^{1-3}$ However, these conventional optical SPPs cannot operate at far-infrared, terahertz (THz), and microwave frequencies where metals are perfect electric conductors. ${ }^{14-26}$ As such, metal sheets with periodic holes or grooves (typically referred to as spoof SPPs), semimetals (such as graphene), semiconductors, and topological insulators have been utilized for SPPs at infrared frequencies and below. ${ }^{24-29}$ Recently, planar and even conformal 2-D metallic line structures have also been proposed to transmit spoof SPPs at THz and microwave frequencies. ${ }^{14-23}$ Their advantages include not only conservation of the intrinsic properties of optical SPPs, e.g., non-diffraction limit, but also low propagation losses, high immunity to electromagnetic couplings, and simple integration with transmission lines, such as co-planar waveguides (CPWs).
Apart from passive SPPs, active SPP devices with electrically tunable properties enable applications in real-time controllable subwavelength circuits, such as switches, attenuators, phase and frequency shifters etc. ${ }^{12,13}$ At optical frequencies liquid plasmonic materials, ferroelectric materials, semiconductors, and P-N diodes have been studied to actively manipulate SPPs. ${ }^{30-36}$ However, their modulation depth is typically less than $20 \%$. To achieve tunable SPPs at microwave frequencies, commercially purchased chips, such as varactor diodes, have been soldered on SPP devices. ${ }^{21-23}$ However, these soldered chips were not monolithically fabricated with the SPP structures. Their device size and integration tolerance have so far limited their operating frequency to below $15 \mathrm{GHz}$. In addition, graphene has recently been investigated to integrate with SPP structures for both tunability and monolithic fabrication over a wider frequency range. ${ }^{37-40}$ However, to the best of our knowledge, the modulation depth of such graphene based active SPP devices only reaches a few percent in experiments. ${ }^{41}$

Here we propose to monolithically fabricate Schottky diodes with a novel subwavelength SPP structure, which enables a significant modulation of transmission, reflection, and absorption for SPPs at millimeter wave frequencies. The proposed device consists of single split-ring resonators (SRRs) on a SPP waveguide and In-Ga-Zn-O (IGZO) Schottky diodes fabricated to bridge the SRR split gaps. The diode can actively tune the conductivity within the split gap so that the resonance magnitude of SRRs and the corresponding attenuation of SPPs are modulated. The characterized continuous modulation depth of transmission and absorption is $40 \%$ and $19 \%$ at $49 \mathrm{GHz}$, respectively, which is, to the best of our knowledge, the record for SPPs. Based on the averaged conductivity derived from the currentvoltage measurement of the IGZO Schottky diode, finite element method (FEM) simulation reveals an ideal modulation range of $67 \%$ for SPP transmission.

\section{NOVEL SPP DEVICE WITH SRRS}

As illustrated in Figure 1a, a novel SPP structure, consisting of two $50-\Omega$ CPWs (I), two CPW-to-SPP waveguide transitions (II), and one SPP waveguide with SRRs (III) is designed on a silicon substrate with a thickness of $200 \mu \mathrm{m}$ and a $100-\mathrm{nm} \mathrm{SiO}_{2}$ layer. Typically, rectangular slots or complementary corrugations are used for spoof SPPs to achieve a spectral cut-off response. ${ }^{16-20}$ Here, single-loop SRRs are proposed to get the cut-off response and band-stop respo- 
(a)

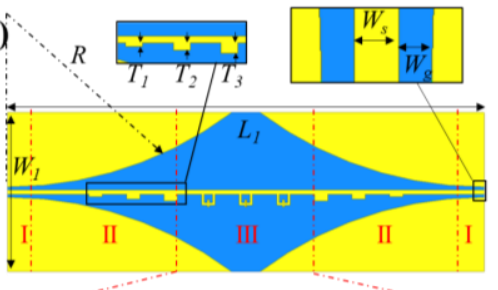

(c)

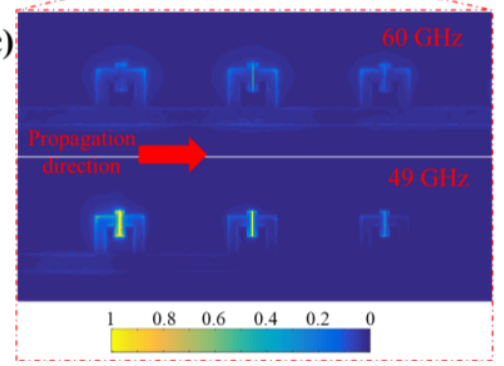

(b)

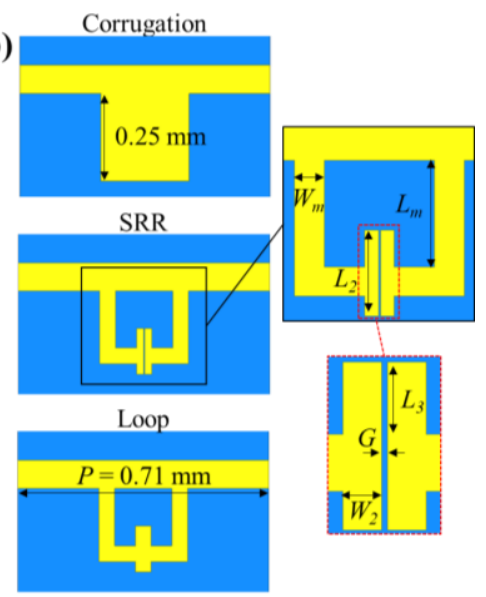

Figure 1. CPW fed SPPs with SRRs: (a) Driven Mode 3-D model; (b) Eigen Mode 3-D models of SPP waveguides with square corrugations, SRRs, and loops in periodic boundary $\left(L_{l}=9 \mathrm{~mm}, W_{l}=3 \mathrm{~mm}, R=7 \mathrm{~mm}, P=0.71 \mathrm{~mm}, W_{s}=80 \mu \mathrm{m}, W_{g}=60 \mu \mathrm{m}, W_{m}=44 \mu \mathrm{m}, L_{2}\right.$ $=130 \mu \mathrm{m}, L_{m}=162 \mu \mathrm{m}, W_{2}=20 \mu \mathrm{m}, G=2.4 \mu \mathrm{m}, L_{3}=56 \mu \mathrm{m}, T_{1}=43 \mu \mathrm{m}, T_{2}=86 \mu \mathrm{m}$, and $T_{3}=130 \mu \mathrm{m}$ ); (c) Simulated norm of electrical fields at the metal-dielectric interface at the resonant $(49 \mathrm{GHz})$ and non-resonant $(60 \mathrm{GHz})$ frequencies.

(a)

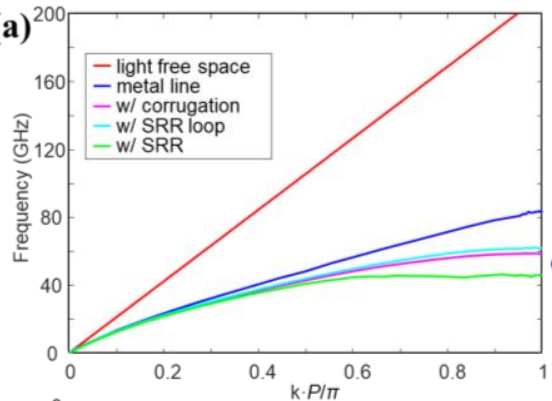

(c)

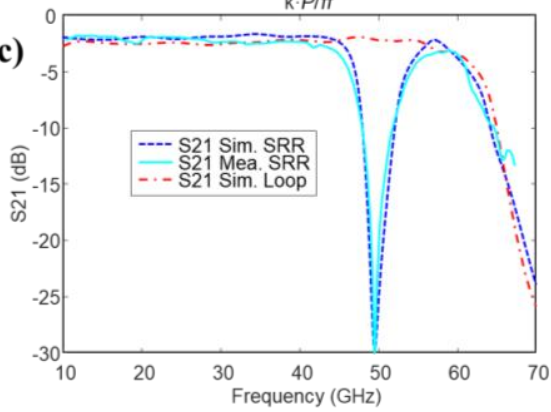

(b)
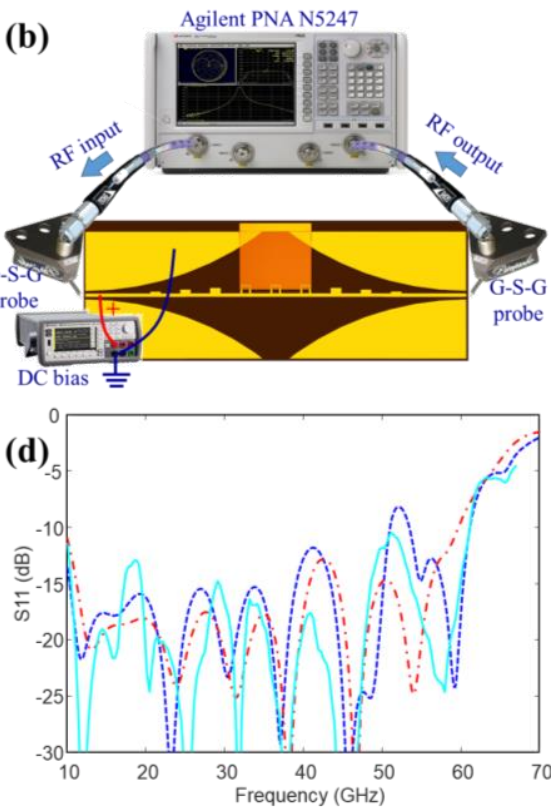

Figure 2. Simulated and measured data and measurement system set-up: (a) Dispersion diagram of the SPP waveguides with corrugations, SRRs, and loops; (b) System set-up with PNA and G-S-G probes, (c) S21 and (d) S11 of the proposed passive device.

nse simultaneously, the latter of which is induced by the resonance of SRRs. The CPW-to-SPP waveguide transition is achieved by flaring out CPW ground electrodes and distributing rectangular corrugations with linearly increased length, whose detailed design can be found in References 17 and 22. Then, SRRs are designed to replace the corrugations without shifting the cut-off frequencies. The resonant frequency of SRRs can be tuned by varying the length $\left(L_{2}\right)$ and width $(G)$ of the gap.

To clarify the SPP cut-off properties, unit cells of SPP waveguides with rectangular corrugations, SRRs, and loops were simulated with periodic boundary using Ansys High Frequency Structural Simulator
(HFSS) Eigen Mode solver. Figure 1b shows their models with a period of $P=0.71 \mathrm{~mm}$, and Figure 2a illustrates their dispersion diagrams. It can be seen that the dispersion curves of the SPP waveguides with corrugations and loops show a similar phenomenon as the optical SPPs and have little discrepancy with each other. ${ }^{1,24}$ The SPP waveguide with SRRs has an additional stop band compared to its counterparts with loops and corrugations. To calculate S-parameters and electric fields, 3-D models of the proposed devices were built in HFSS Driven Mode solver and simulated in a radiation boundary with two wave ports as the input and output for the CPWs. The optimized dimensions of a SPP device with its stop band centered at $49 \mathrm{GHz}$ are listed in the caption of Figure 1. The proposed 
device addresses a miniaturized size of $0.052 \lambda_{0} \times 0.278 \lambda_{0}$ at its resonant frequency. As illustrated in Figure 1c, SPPs attenuate significantly at the band-stop frequencies, e.g., $49 \mathrm{GHz}$, due to the strong resonance of the SRRs, and propagate with little attenuation at the non-resonant frequencies, e.g., $60 \mathrm{GHz}$.

Agilent programmable network analyzer (PNA) N5247 was used to characterize the proposed passive and active devices, and the system set-up is depicted in Figure 2b. 50- $\Omega$ ground-signal-ground (GS-G) probes integrated with PNA were first calibrated with CS-5 onwafer calibration chip, and then launched on the two CPWs for signal input and output. The measured S-parameters of the passive device are illustrated in Figure 2c and 2d, showing good agreement with the simulated ones. The device has a stop band centered at $49 \mathrm{GHz}$ with a Q-factor of 93, and its maximum bandstop attenuation is $-31 \mathrm{~dB}$. The propagation attenuation is less than $2.2 \mathrm{~dB}$ in the pass band, which can be mainly attributed to the insertion loss of the CPW-toSPP waveguide transitions (Supporting information, Figure S1).

\section{MODULATION WITH SCHOTTKY DIODES}

Inspired by tunable metamaterials with gallium arsenide (GaAs) Schottky diodes ${ }^{42,43}$ for the first time Schottky diodes are proposed to actively manipulate SPPs here, as shown in Figure 3a. The diodes are fabricated using IGZO oxide semiconductor to bridge the split gaps of SRRs. Compared to GaAs, IGZO can be sputtered onto various substrates, including the flexible ones. ${ }^{44-48}$ The Schottky and ohmic contacts of the diode are the SRRs and CPW grounds, respectively. To minimize the impact to SPP propagation, the ohmic contact is designed around $1.2 \mathrm{~mm}$ away from the SRRs (Supporting information, Figure S1c).The corresponding fabrication steps are illustrated in Figure 3b. First, the signal electrode (Schottky contact) was fabricated by conventional photolithography and electron-beam deposition of $10 \mathrm{~nm}$ titanium, $300 \mathrm{~nm}$ gold, and $50 \mathrm{~nm}$ palladium. Next, a 750-nm IGZO film was grown by using sputtering at room temperature. Finally, the ground electrode (ohmic contact) was deposited by using $10-\mathrm{nm}$ titanium and $300-\mathrm{nm}$ gold. In this case, a Schottky junction is formed between the IGZO film and the signal electrode, as shown in Figure 3c, which could be demonstrated by the DC current-voltage measurement (Supporting information, Figure S2a).
The equivalent circuit model of the proposed approach is a $R L C$ resonant circuit depicted in Figure 3d, where $L$ is the inductance of the ring loop, $C$ is the capacitance of the split gap, and $R_{g}$ represents the variable attenuation due to the substrate free carrier absorption within the split gap. At the resonant frequencies, e.g., $49 \mathrm{GHz}$, SPPs are attenuated by the SRR resonance significantly, as illustrated in Figure 1c. At zero bias, the IGZO film in the split gap is depleted so that the SRRs resonate normally and thus shows a perfect band-stop response. This initial state corresponds to the blue curve in Figure 2c. As the forward bias increases, the free carriers with increasing density short out the capacitor gradually, as illustrated in Figure 3d. Thus, the SRR resonance becomes subdued, and the SRR with Schottky diode works more and more like the metallic loop illustrated in Figure $1 \mathrm{~b}$, which leads to smaller attenuation for SPP propagation. Ideally, the ultimate state is the red curve in Figure 2c, and the active modulation curve should locate between the blue and red curves.

The measured S-parameters of the active device are illustrated in Figure $4 \mathrm{a}$ and $4 \mathrm{~b}$, showing a large modulation of both transmission and reflection. The transmission was modulated from -31 to $-6.9 \mathrm{~dB}$ at $49 \mathrm{GHz}$ with a varying forward bias, which corresponds to a modulation depth of as high as $40 \%$ in Figure 5a. The maximum forward bias applied is $46 \mathrm{~V}$, which is limited by the protect voltage of the PNA bias-Tee. Note that little spectral shifting of the resonant frequency was observed in our measurement. This phenomenon can be explained by the quasi-constant capacitance of the Schottky diode with a forward bias (Supporting information, Figure S2c), which remains fairly stable as a function of frequency. ${ }^{44}$ To further reveal the tunability of the proposed device, the absorption and differential transmission defined as $\Delta T / T_{0}$ are plotted in Figure $4 \mathrm{c}$ and $4 \mathrm{~d}$, respectively ( $\Delta T$ is the transmission discrepancy, and $T_{0}$ is the transmission at zero bias). A dramatic modulation range of $19 \%$ is demonstrated for the SPP absorption. With respect to the zero-bias case, the absorption first increases at a bias of $5 \mathrm{~V}$, then decreases due to the increasing conductivity within the SRR split gap as the bias further enlarges. As can be seen from Figure 4d, the differential transmission increases continuously as the applied voltage enlarges. At a bias of $46 \mathrm{~V}, \Delta T / T_{0}$ is as high as 257 , which to the best of our knowledge is the highest for the active SPP devices. 

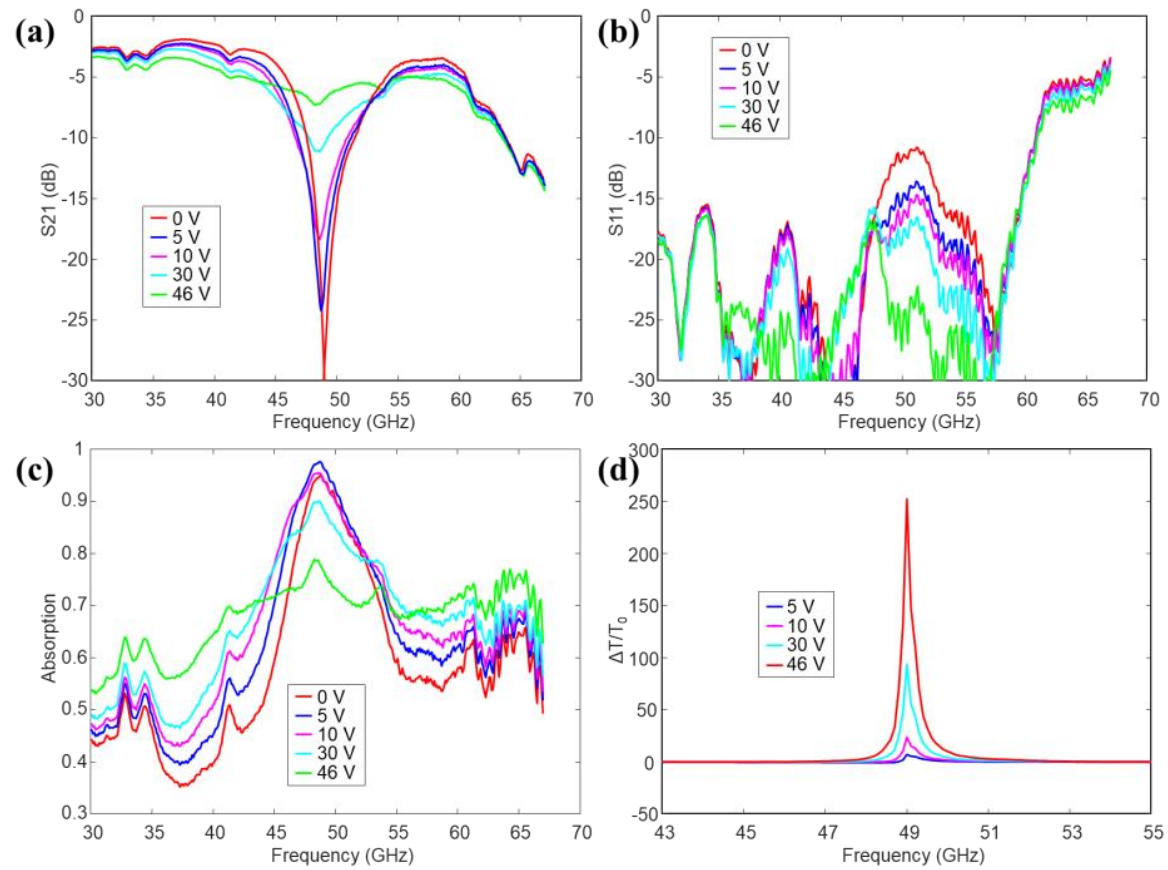

Figure 4. Modulation performance of the active SPP device at different forward bias: frequency-dependent (a) Transmission, (b) Reflection and (c) Absorption of the device at different bias, (d) Differential transmission of the device with respect to the transmission at zero bias.
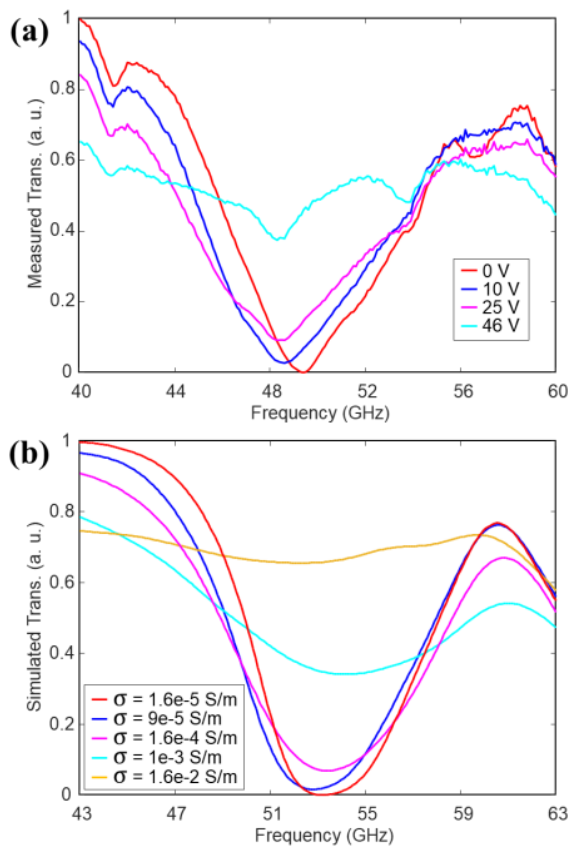

Figure 5. (a) Measured and (b) Simulated normalized transmission of the active SPP device.

To reveal the ideal modulation range, averaged conductivity of the IGZO film was extracted from the DC current-voltage measurement of the Schottky diode (Supporting information, Figure S2b), which changes from $7.9 \times 10^{-6}$ to $0.23 \mathrm{~S} / \mathrm{m}$ with bias sweeping. Thus, a sheet with different conductivities was designed for IGZO to bridge the
SRR gap in full-wave HFSS simulation. Figure 5 depicts the normalized transmission curves extracted from the measured and simulated S-parameters (Supporting information, Figure S3), whose discrepancy can be mainly attributed to the structure difference between the simulation model and fabricated device. Although surface roughness, fabrication tolerance, and spatial non-uniformity of conductivity in IGZO film are not considered in the simulation, the simulated and measured data still show reasonable agreement. As the orange curve depicted in Figure 5b, the device has little bandstop attenuation at 49 $\mathrm{GHz}$ with a conductivity of $0.016 \mathrm{~S} / \mathrm{m}$, which can be easily obtained with a higher forward DC bias. In this case, the ideal modulation range of the transmission and absorption is $67 \%$ and $32 \%$, respectively, and the corresponding differential transmission is as high as 573. Due to the large distance between the ohmic and Schottky contacts, the applied bias here is higher than several reported works. ${ }^{35}$, ${ }^{36}$ However, the applied bias is dominantly dropped over the long IGZO channel and the power dissipation is distributed over a wide range. Several devices have been tested successfully without any breakdown in the aforementioned voltage range.

\section{CONCLUSION}

Monolithic oxide-semiconductor based Schottky diodes have been demonstrated to successfully manipulate SPPs with subwavelength size, achieving a remarkable modulation depth of $19 \%$ and $40 \%$ for the absorption and transmission at millimeter wave frequencies, respectively. This approach is highly practical because oxide semiconductors can be deposited on large-area and/or flexible substrates at a low cost using sputtering method. The demonstrated tunable SPP devices may be scaled to other microwave and $\mathrm{THz}$ frequencies and/or integrated further with other components for more functional monolithic integrated circuits.

\section{ASSOCIATED CONTENT}




\section{Supporting Information}

(1) HFSS model of CPW-to-spoof SSP transitions and the extracted insertion loss; (2) DC response of the proposed Schottky diode; (3) simulated modulation of the active spoof SPP device with an IGZO film of different conductivity. (PDF)

\section{Fabrication}

The substrate was sequentially cleaned in an ultrasonic bath with decon, de-ionized water, acetone, and ethanol, and then dried by $\mathrm{N}_{2}$. The signal electrodes of CPWs and SPP waveguide (Ti $10 \mathrm{~nm} / \mathrm{Au}$ $300 \mathrm{~nm} / \mathrm{Pd} 50 \mathrm{~nm}$ ) were defined using the laser direct writing (LDW), deposited with electron-beam evaporation, and patterned with lift-off process. The Pd layer was treated with oxygen plasma (PDC-21G, 30min) for oxidation to ensure good oxygen stoichiometry of the contact interface before IGZO deposition. Then, a 750nm-thick amorphous IGZO layer was deposited with a target of In:Ga: $\mathrm{Zn}=1: 1: 1$ in atomic ratio (Lesker Co.) and a mixed gas of $97.5 \%$ argon and $2.5 \%$ oxygen by using radio-frequency (RF) magnetron sputtering at room temperature. The RF sputtering power was $70 \mathrm{~W}$ and the working pressure was 3.63 mTorr. Finally, the ground electrodes of CPWs and DC bias (Ti $10 \mathrm{~nm} / \mathrm{Au} 300 \mathrm{~nm}$ ) were also defined by LDW, deposited with electron-beam evaporation, and patterned with lift-off as the top ohmic contact.

\section{AUTHOR INFORMATION}

\section{Corresponding Author}

Email: wangqingpu@sdu.edu.cn, songam@sdu.edu.cn

\section{Author Contributions}

Y. Zhang, Q. Wang, and A. Song contributed equally to this work. Notes

The authors declare no competing financial interests.

\section{ACKNOWLEDGMENT}

The authors would like to acknowledge the National Key Research and Development Program of China (Grant Nos. 2016YFA0301200 and 2016YFA0201800), the National Natural Science Foundation of China (Grant Nos. 61701283 and 11404115), the European Commission through the FP7 ROOTHz project (Grant No. 243845), Engineering and Physical Sciences Research Council (EPSRC) (Grant No. EP/N021258/1), China Postdoctoral Science Foundation funded project (Grant Nos. 2018T110689, 2017M622201, and 2016M590634), the Key Research and Development Program of Shandong Province (Grant Nos. 2017GGX10121 and 2017GGX10111), Postdoctoral Innovation Program of Shandong Province (Grant No. 20171006), and the Fundamental Research Funds of Shandong University (Grant No. 2018TB002 and 2016WLJH44) for supporting this work.

\section{REFERENCES}

(1) Hibbins, A. P.; Evans, B. R.; Sambles, J. R. Experimental verification of designer surface plasmons. Science 2005, 308, 670-672.

(2) Berini, P.; De Leon, I. Surface plasmon-polariton amplifiers and lasers. Nat. Photonics 2011, 6, 16-24.

(3) Novotny, L.; van Hulst, N. Antennas for light. Nat. Photonics 2011, 5, 83-90

(4) Dong, S.; Zhang, K.; Yu, Z.; Fan, J. A. Electrochemically programmable plasmonic antennas. ACS Nano 2016, 10, 6716-6724.

(5) Li, X.; Zhu, J.; Wei, B. Hybrid nanostructures of metal/two-dimensional nanomaterials for plasmon-enhanced applications. Chem. Soc. Rev. 2016, 45, 3145-3187.
(6) Gramotnev, D. K.; Bozhevolnyi, S. I. Plasmonics beyond the diffraction limit. Nat. Photonics 2010, 4, 83-91.

(7) Tang, H.-H.; Liu, P. K. Terahertz far-field superresolution imaging through spoof surface plasmons illumination. Optics Lett. 2015, 40 (24), 5822-5825.

(8) Ren, H.; Li, X.; Zhang, Q.; Gu, M. On-chip non-interference angular momentum multiplexing of broadband light. Science 2016, 352, 805-809.

(9) Chen, X.; Fan, W. Ultrasensitive terahertz metamaterial sensor based on spoof surface plasmon. Sci. Rep. 2017, 7, 2092.

(10) Choo, H.; Kim, M.-K.; Staffaroni, M.; Seok, T. J.; Bokor, J.; Cabrini, S.; Schuck, P. J.; Wu, M. C.; Yablonovitch, E. Nanofocusing in a metalinsulator-metal gap plasmon waveguide with a three-dimensional linear taper. Nat. Photonics 2012, 6, 838-844.

(11) Jang, Y. H.; Jang, Y. J.; Kim, S.; Quan, L. N.; Chung, K.; Kim, D. H. Plasmonic solar cells: from rational design to mechanism overview. Chem. Rev. 2016, 116 (24), 14982-15034.

(12) Barnes, W. L.; Dereux, A.; Ebbesen, T. W. Surface plasmon subwavelength optics. Nature 2003, 424, 824-830.

(13) Vakil, A.; Engheta, N. Transformation optics using graphene. Science 2011, 332, 1291-1294.

(14) Gan, Q.; Zhan, F.; Ding, Y. J.; Bartoli, F. J. Ultrawide-bandwidth slow-light system based on $\mathrm{THz}$ plasmonic graded metallic grating structures. Phys. Rev. Lett. 2008, 100, 256803.

(15) Wang, K.; Mittleman, D. M. Metal wires for terahertz wave guiding. Nature 2004, 432, 376-379.

(16) Chen, L.; Wei, Y.; Zang, X.; Zhu, Y.; Zhuang, S. Excitation of dark multipolar plasmonic resonances at terahertz frequencies. Sci. Rep. 2016, 6, 22027.

(17) Liu, X.; Feng, Y.; Zhu, B.; Zhao, J.; Jiang, T. Backward spoof surface wave in plasmonic metamaterial of ultrathin metallic structure. Sci. Rep. 2016, 6, 20448.

(18) Ma, H. F.; Shen, X.; Cheng, Q.; Jiang, W. X.; Cui, T. J. Broadband and high-efficiency conversion from guided waves to spoof surface plasmon polaritons. Laser \& Photonics Reviews 2014, 8, 146-151.

(19) Zhang, H. C.; Cui, T. J.; Zhang, Q.; Fan, Y.; Fu, X. Breaking the challenge of signal integrity using time-domain spoof surface plasmon polaritons. ACS Photonics 2015, 2, 1333-1340.

(20) Pan, B. C.; Liao, Z.; Zhao, J.; Cui, T. J. Controlling rejections of spoof surface plasmon polaritons using metamaterial particles. Opt. Express 2014, 22, 13940-13950.

(21) Xu, J.; Zhang, C.; Tang, W.; Guo, J.; Qian, C.; Li, W. Transmissionspectrum-controllable spoof surface plasmon polaritons using tunable metamaterial particles. Appl. Phys. Lett. 2016, 108, 191906.

(22) Zhang, H. C.; Cui, T. J.; Xu, J.; Tang, W.; Liu, J. F. Real-time controls of designer surface plasmon polaritons using programmable plasmonic metamaterial. Adv. Mater. Technol. 2017, 2, 1600202.

(23) Zhang, H. C.; Fan, Y.; Guo, J.; Fu, X.; Cui, T. J. Second-harmonic generation of spoof surface plasmon polaritons using nonlinear plasmonic metamaterials. ACS Photonics 2015, 3, 139-146.

(24) Pendry, J. B.; Martin-Moreno, L.; Garcia-Vidal, F. J. Mimicking surface plasmons with structured surfaces. Science 2004, 305, 847-848.

(25) Williams, C. R.; Andrews, S. R.; Maier, S. A.; FernandezDominguez, A. I.; Martin-Moreno, L.; Garcia-Vidal, F. J. Highly confined guiding of terahertz surface plasmon polaritons on structured metal surfaces. Nat. Photonics 2008, 2, 175-179.

(26) Law, S.; Yu, L.; Rosenberg, A.; Wasserman, D. All-semiconductor plasmonic nanoantennas for infrared sensing. Nano Lett. 2013, 13, 45694574.

(27) Grigorenko, A. N.; Polini, M.; Novoselov, K. S. Graphene plasmonics. Nat. Photonics 2012, 6, 749-758.

(28) Lu, H.; Li, Y.; Yue, Z.; Mao, D.; Zhao J. Topological insulator based Tamm plasmon polaritons. APL Photonics 2019, 4, 040801.

(29) Yuan, J.; Ma, W.; Zhang, L.; Lu, Y.; Zhao, M.; Guo, H.; Zhao, J.; Yu, W.; Zhang, Y.; Zhang, K.; Hoh, H. Y.; Li, X.; Loh, K. P.; Li, S.; Qiu, C.-W.; Bao, Q. Infrared nanoimaging reveals the surface metallic plasmons in topological insulator. ACS Photonics 2017, 4, 3055-3062.

(30) Vivekchand, S. R. C.; Engel, C. J.; Lubin, S. M.; Blaber, M. G.; Zhou, W.; Suh, J. Y.; Schatz, G. C.; Odom, T. W. Liquid plasmonics: manipulating surface plasmon polaritons via phase transitions. Nano Lett. 2012, 12, 4324-4328.

(31) Rudé, M.; Simpson, R. E.; Quidant, R.; Pruneri, V.; Renger, J. Active control of surface plasmon waveguides with a phase change material. ACS Photonics 2015, 2, 669-674. 
(32) Dicken, M. J.; Sweatlock, L. A.; Pacifici, D.; Lezec, H. J.; Bhattacharya, H.; Atwater, A. Electrooptic modulation in thin film barium titanate plasmonic interferometers. Nano Lett. 2008, 8 (11), 4048-4052.

(33) Pacifici, D.; Lezec, H. J.; Atwater, H. A. All-optical modulation by plasmonic excitation of CdSe quantum dots. Nat. Photonics 2007, 1, 402406.

(34) Moilanen, A. J. Active control of surface plasmon-emitter strong coupling. ACS Photonics 2018, 5, 54-64.

(35) Dong, Z.; Vinnakota, R. K.; Briggs, A. F.; Nordin, L.; Bank, S. R.; Genov, D. A.; Wasserman, D. Electrical modulation of degenerate semiconductor plasmonic interfaces. J. Appl. Phys. 2019, 126, 043101.

(36) Vinnakota, R.; Genov, D. A. Active control of charge density waves at degenerate semiconductor interfaces. Sci. Rep. 2017, 7, 10778.

(37) Shi, B.; Cai, W.; Zhang, X.; Xiang, Y.; Zhan, Y.; Geng, J.; Ren, M.; $\mathrm{Xu}$, J. Tunable band-stop filters for graphene plasmons based on periodically modulated graphene. Sci. Rep. 2016, 6, 26796.

(38) Lu, H.; Gan, X.; Mao, D.; Jia, B.; Zhao, J. Flexibly tunable highquality-factor induced transparency in plasmonic systems. Sci. Rep. 2018, 8,1558 .

(39) Lu, H.; Zeng, C.; Zhang, Q.; Liu, X.; Hossain, M. M.; Reineck, P.; $\mathrm{Gu}, \mathrm{M}$. Graphene-based active slow surface plasmon polaritons. Sci. Rep. 2015, 5, 8443.

(40) Lu, H.; Gan, X.; Mao, D.; Zhao, J. Graphene-supported manipulation of surface plasmon polaritons in metallic nanowaveguides. Photonics Research 2017, 5, 162-167.
(41) Gao, W.; Shi, G.; Jin, Z.; Shu, J.; Zhang, Q.; Vajtai, R.; Ajayan, P. M.; Kono, J.; Xu, Q. Excitation and active control of propagating surface plasmon polaritons in graphene. Nano Lett. 2013, 13, 3698-3702.

(42) Chen, H.-T.; Padilla, W. J.; Zide, J. M. O.; Gossard, A. C.; Taylor, A. T.; Averitt, R. D. Active terahertz metamaterial devices. Nature 2006, $444,597-600$

(43) Chen, H.-T.; Padilla, W. J.; Cich, M. J.; Azad, A. K.; Averitt, R. D.; Taylor, A. J. A metamaterial solid-state terahertz phase modulator. Nat. Photonics 2009, 3, 148-151.

(44) Zhang, J.; Li, Y.; Zhang, B.; Wang, H.; Xin, Q.; Song, A. Flexible indium-gallium-zinc-oxide Schottky diode operating beyond $2.45 \mathrm{GHz}$. Nat. Commun. 2015, 6, 7561.

(45) Hosono, H. How we made the IGZO transistor. Nature Electronics $2018,1,428$.

(46) Cai, W.; Zhang, J.; Wilson, J.; Ma, X.; Wang, H.; Zhang, X.; Xin, Q.; Song, A. Oxide-based electric-double-layer thin-film transistors on a flexible substrate. IEEE Electron Device Lett. 2017, 38, 1680-1683.

(47) Yang, J.; Wang, Y.; Li, Y.; Yuan, Y.; Hu, Z.; Ma, P.; Zhou, L.; Wang, Q.; Song, A.; Xin, Q. Highly optimized complementary inverters based on $\mathrm{p}-\mathrm{SnO}$ and $\mathrm{n}-\mathrm{InGaZnO}$ with high uniformity. IEEE Electron Device Lett. 2018, 39, 516-519.

(48) Arora, H.; Malinowski, P. E.; Chasin, A.; Cheyns, D.; Steudel, S.; Schols, S.; Heremans, P. Amorphous indium-gallium-zinc-oxide as electron transport layer in organic photodetectors. Appl. Phys. Lett. 2015, 116 143301.

Graphic entry for the Table of Contents (TOC)

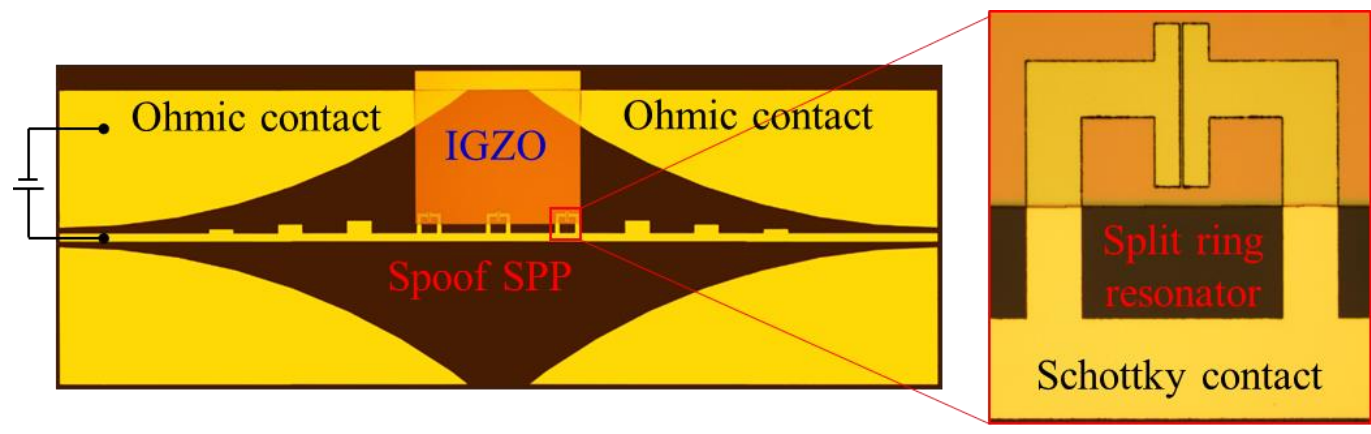

Keywords: Surface plasmon polariton, Schottky diode, active modulation, split-ring resonator, In-Ga-Zn-O 